\title{
Zoledronic acid cooperates with a cyclooxygenase-2 inhibitor and gefitinib in inhibiting breast and prostate cancer
}

\author{
Davide Melisi ${ }^{1}$, Rosa Caputo ${ }^{1}$, Vincenzo Damiano ${ }^{1}$, Roberto Bianco ${ }^{1}$, \\ Bianca Maria Veneziani ${ }^{3,4}$, A Raffaele Bianco ${ }^{1,4}$, Sabino De Placido ${ }^{1,4}$, \\ Fortunato Ciardiello ${ }^{2,4}$ and Giampaolo Tortora ${ }^{1,4}$
}

\footnotetext{
${ }^{1}$ Cattedra di Oncologia Medica, Dipartimento di Endocrinologia e Oncologia Molecolare e Clinica, Università di Napoli Federico II, Via S. Pansini 5, 80131 Naples, Italy

${ }^{2}$ Cattedra di Oncologia Medica, Dipartimento Medico-Chirurgico di Internistica Clinica e Sperimentale 'F Magrassi e A Lanzara', Seconda Università di Napoli, Via S. Pansini 5, 80131 Naples, Italy

${ }^{3}$ Dipartimento di Biologia e Patologia Cellulare e Molecolare, Università di Napoli Federico II, Via S. Pansini 5, 80131 Naples, Italy ${ }^{4}$ Oncotech, Via S. Pansini 5, 80131 Naples, Italy
}

(Requests for offprints should be addressed to G Tortora, Cattedra di Oncologia Medica, Dipartimento Endocrinologia e Oncologia Molecolare e Clinica, Università di Napoli Federico II, Via S. Pansini 5, 80131 Napoli, Italy; Email: gtortora@ unina.it)

\begin{abstract}
Biphosphonates (BPs) are widely used to inhibit osteoclastic activity in malignant diseases such as bone metastatic breast and prostate carcinoma. Recent studies reported that BPs could also cause a direct antitumor effect, probably due to their ability to interfere with several intracellular signalling molecules. The enzyme cyclooxygenase-2 (COX-2) and the epidermal growth factor receptor (EGFR) play an important role in the control of cancer cell growth and inhibitors of COX-2 and EGFR have shown antitumor activity in vitro and in vivo in several tumor types. We, and others, have previously shown that EGFR and COX-2 may be directly related to each other and that their selective inhibitors may have a cooperative effect. In the present study we have evaluated the combined effect of zoledronic acid, the most potent nitrogen-containing BP, with the COX-2 inhibitor SC-236 and the selective EGFR-tyrosine kinase inhibitor gefitinib, on breast and prostate cancer models in vitro and in xenografted nude mice. We show that combination of zoledronic acid with SC-236 and gefitinib causes a cooperative antitumor effect accompanied by induction of apoptosis and regulation of the expression of mitogenic factors, proangiogenic factors and cell cycle controllers both in vitro and in xenografted nude mice. The modulatory effect on protein expression and the inhibitory effect on tumor growth is much more potent when the three agents are used together. Since studies are ongoing to explore the antitumor effect of zoledronic acid, our results provide new insights into the mechanism of action of these agents and a novel rationale to translate this feasible combination treatment strategy into a clinical setting.
\end{abstract}

Endocrine-Related Cancer (2005) 12 1051-1058

\section{Introduction}

The increased understanding of intracellular signaling mechanisms has highlighted the role played in the control of cancer cell growth and spread by several critical proteins, now regarded as potentially relevant targets for therapeutic intervention. A pivotal role is played by the epidermal growth factor receptor (EGFR), which is overactivated in a wide variety of epithelial cancers and triggers a cascade of signals affecting multiple cellular functions (Ciardiello \& Tortora 2001, Mendelsohn \& Baselga 2003). Several selective EGFR inhibitors have successfully entered clinical evaluation - including the tyrosine kinase inhibitors gefitinib and erlotinib, and the monoclonal antibody cetuximab (Mendelsohn \& Baselga 2003). Among the proteins activated by EGFR, the small GTPases of the Ras and Rho families have been reported as dysregulated in different types of cancer (Sinensky 2000). Recent studies have also indicated 
that cyclooxygenase-2 (COX-2) - an enzyme that catalyzes the formation of prostaglandins (PGs) and is involved in cancer cell proliferation, angiogenesis and apoptosis (Turini \& DuBois 2002) - is transcriptionally activated following EGFR activation via Ras-mediated signalling (Smith et al. 2000). Moreover, we have demonstrated that cancer cells that have acquired resistance to EGFR inhibitors gefitinib and cetuximab, overexpress COX-2 and vascular endothelial growth factor (VEGF) as escape pathways to EGFR blockade (Ciardiello et al. 2004). For these reasons COX-2 is also considered a potential target for anticancer therapy and several selective inhibitors are currently under clinical evaluation. In this regard, we have demonstrated that combined blockade of EGFR and COX-2 by gefitinib and the COX-2 inhibitor SC236, an analog of celecoxib with antiproliferative and antiangiogenic properties (Masferrer et al. 1999), causes a cooperative antitumor and antiangiogenic effect in vitro and in vivo in human cancer models (Tortora et al. 2003).

These data suggest that the functional interactions among different signalling proteins may sustain cancer cell growth and dissemination, and that their combined blockade may produce a cooperative effect overcoming the possible occurrence of resistance.

For this purpose we have combined the EGFR inhibitor gefitinib and the COX-2 inhibitor SC-236 with the novel biphosphonate (BP) zoledronic acid (ZA). BPs, expecially those containing a tertiary amine within a ring structure, such as ZA, are potent inhibitors of osteoclast-mediated bone resorption and are widely used for the treatment of breast and prostate carcinoma bone metastases (Lacerna \& Hohneker 2003). Recent studies suggest that ZA also has direct antitumor effects: inhibition of tumor cell proliferation, adhesion, invasion and angiogenesis; promotion of apoptosis; local reduction of growth factors; and cytokines release (Green 2003). Although the precise mode of action of nitrogen-containing BPs is still not fully understood, one possible mechanism is based on their ability to impair post-translational prenylation of Ras and Rho (Sinensky 2000).

We have evaluated the antitumor activity in vitro and in vivo of $\mathrm{ZA}$, gefitinib and SC-236 in human breast and prostate cancer cell models.

\section{Materials and Methods}

\section{Materials}

Clinical grade gefitinib was provided by AstraZeneca, ZA (1-hydroxy-2-(1H-imidazole-1-yl)ethylidene-bisphosphonic acid) was obtained in the form of its hydrated disodium salt from Novartis Pharma AG and SC-236 was provided by Pfizer (Groton, CT, USA).

\section{Cell lines}

PC3 human prostate and ZR-75-1 human breast cancer cell lines were obtained from the American Type Culture Collection (Manassas, VA, USA). PC3 and ZR-75-1 cells were maintained in RPMI 1640 and Dulbecco's modified Eagle's medium (DMEM) respectively, supplemented with $10 \%$ fetal bovine serum, penicillin $(100 \mathrm{UI} / \mathrm{ml})$, streptomycin $(100 \mu \mathrm{g} / \mathrm{ml})$ and $4 \mathrm{mM}$ glutamine (ICN, Irvine, UK) in a humidified atmosphere of $95 \%$ air and $5 \% \mathrm{CO}_{2}$ at $37{ }^{\circ} \mathrm{C}$.

\section{Growth in soft agar and analysis of combination index $(\mathrm{Cl})$}

On day 0 , cells ( 10 cells/well) were suspended in $0.5 \mathrm{ml}$ of $0.3 \%$ Difco Noble agar (Difco, Detroit, MI, USA) supplemented with complete culture medium. This suspension was layered over $0.5 \mathrm{ml}$ of $0.8 \%$ agar-medium base layer in 24-multiwell cluster dishes (Becton Dickinson, Lincoln Park, NJ, USA) and treated on days 0,2 and 4 with the following concentrations of drugs: ZA, from 0.5 to $50 \mu \mathrm{M}$; SC236,1 and $2.5 \mu \mathrm{M}$; gefitinib, $0.5 \mu \mathrm{M}$. After 10-14 days, cells were stained with nitro blue tetrazolium (Sigma), and colonies $>0.05 \mathrm{~mm}$ were counted. Growth inhibition results are expressed as the percentage of the number of colonies developed in each treatment well as compared with the absolute number of colonies developed in the untreated control group.

Assessment of synergy was performed following the method described by Chou and Talalay (1984) and using the Calcusyn software program (Biosoft, Cambridge, UK). According to this method, CI values of $<1,1$ and $>1$ indicate synergy, additivity and antagonism respectively.

\section{Apoptosis in cultured cells}

The induction of apoptosis was determined by the Cell Death Detection ELISA Plus Kit, which detects cytosolic histone-associated DNA fragments (Roche, Molecular Biochemicals, Mannheim, Germany). Briefly, PC3 and ZR-75-1 cells $\left(5 \times 10^{4}\right.$ cells/dish) were seeded into $35-\mathrm{mm}$ dishes. Cells treated on days 1 to 5 with ZA $(5 \mu \mathrm{M})$ and SC-236 $(2.5 \mu \mathrm{M})$, and gefitinib $(1 \mu \mathrm{M})$ alone or in combination, on day 6 were washed once with PBS; then $0.2 \mathrm{ml}$ of lysis buffer was added. After a 30-min incubation, the supernatant was recovered and assayed for DNA fragments as recommended by the manufacturer. Each treatment was 
performed in quadruplicate. The total number of cells was measured with a hemocytometer in additional plates receiving an identical treatment. The values resulting from readings of absorbance at A405 $\mathrm{nm}$ were normalized for cell number, and the ratio of the absorbance of treated cells to that of untreated cells was defined as the apoptotic index (AI).

\section{Western blot analysis}

Total cell lysates were obtained from either homogenized PC3 xenograft tumor specimens or from PC-3 and ZR-75-1 cells cultured in vitro. PC-3 and ZR-75-1
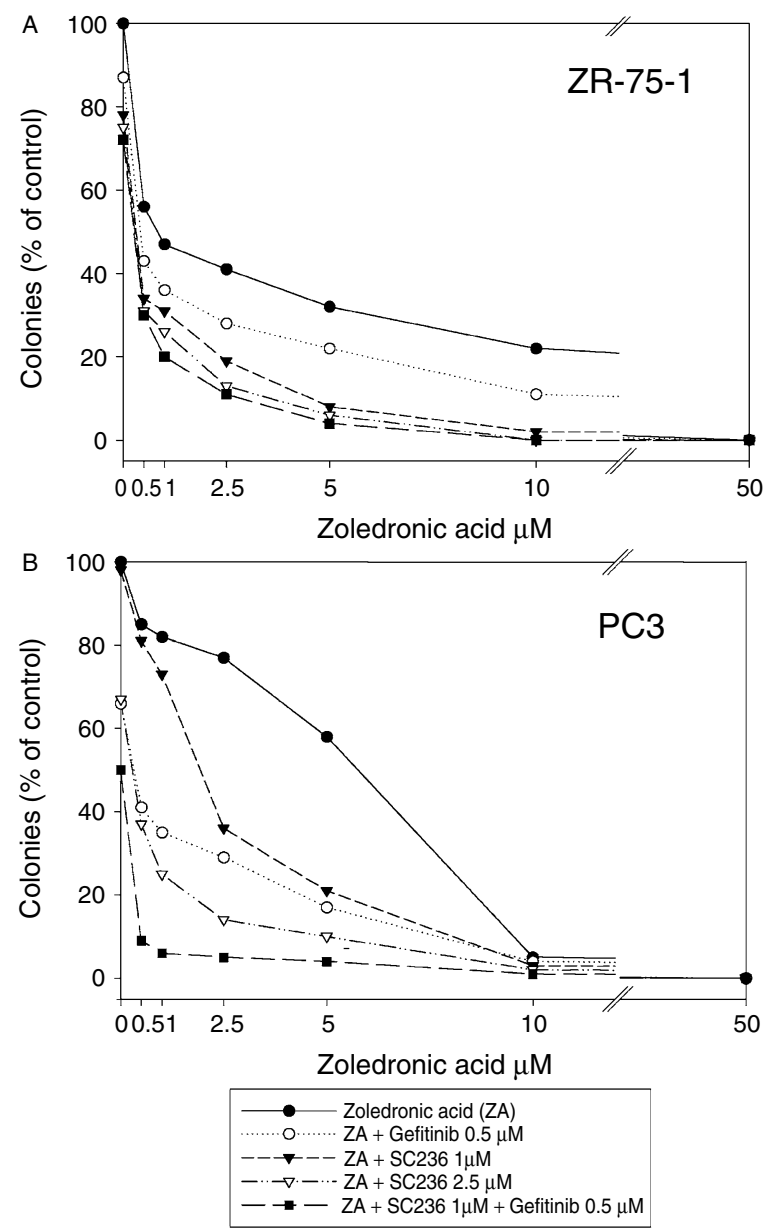

Figure 1 Effect of ZA, SC-236 and gefitinib, alone and in combination, on the soft agar growth of (A) ZR-75-1 and (B) PC3 cells. Growth inhibition results are expressed as the percentage of the number of colonies developed in each of the different treatment wells compared with the absolute number of colonies developed in the untreated control group, which was 85 in ZR-75-1 and 115 in PC3 cells respectively. Data represent the average of at least two different experiment run in triplicate. cells were treated on days 1 to 5 with ZA $(5 \mu \mathrm{M})$ and SC-236 $(2.5 \mu \mathrm{M})$, and gefitinib $(1 \mu \mathrm{M})$ alone or in combination. On day 6 the protein extracts were resolved by $4-15 \%$ SDS-PAGE and probed with antihuman polyclonal COX-2, monoclonal phosphorylated mitogen-activated protein kinase (pMAPK), monoclonal p27, monoclonal Ras and VEGF antibodies (Santa Cruz Biotechnology, Santa Cruz, CA, USA). Immunoreactive proteins were visualized by enhanced chemiluminescence (Amersham), as described previously (Tortora et al. 1997).

\section{PC3 xenografts in nude mice}

The 5- to 6-week-old Balb/cAnNCrlBR athymic (nu+/ $\mathrm{nu}+$ ) mice were purchased from Charles River Laboratories (Milan, Italy). The research protocol was approved, and mice were maintained in accordance with institutional guidelines of the University of Naples Animal Care and Use Committee. Mice were acclimatized to the University of Naples Medical School Animal Facility for 1 week before they received injections of cancer cells: 10 PC3 human prostate cancer cells were resuspended in $200 \mu$ l Matrigel (Collaborative Biomedical Products, Bedford, MA, USA) and injected s.c. in mice. After 7 days, when well-established tumors of $\sim 0.2 \mathrm{~cm}$ were detected, mice were randomized to receive different treatments. Groups of 8 mice were treated i.p. with ZA $(400 \mathrm{mg} /$ $\mathrm{kg}$ per day, days 2 and 4 of each week), SC-236 (12 mg/ $\mathrm{kg}$ per day, days 1 to 5 of each week) and gefitinib $(150 \mathrm{mg} / \mathrm{kg}$ per day, days 1 to 5 of each week) for 4 weeks. Tumor volume was measured using the formula: $\pi / 6 \times$ larger diameter $\times(\text { smaller diameter })^{2}$; as reported previously (Ciardiello et al. 1996).

\section{Results \\ Effect of combined treatment on cancer cell growth in vitro}

As shown in Fig. 1A and B, we first evaluated the antitumor activity of these drugs on the soft agar growth in vitro of PC3 prostate cancer and ZR-75-1 breast cancer cells. ZA caused a dose-dependent growth inhibition of both tested cells, resulting in approximately $43 \%$ inhibition at $0.5 \mu \mathrm{M}$ and $77 \%$ at $10 \mu \mathrm{M}$ in ZR-75-1 cells, while the same doses produced inhibition of approximately 17 and $95 \%$ respectively in PC3 cells. Growth inhibition increased when ZA was combined with either gefitinib $(0.5 \mu \mathrm{M})$ or SC-236 (1.0 or $2.5 \mu \mathrm{M})$, and the most potent effects were seen when all three agents were combined in both cell lines. To better evaluate the interaction and the possible 
A
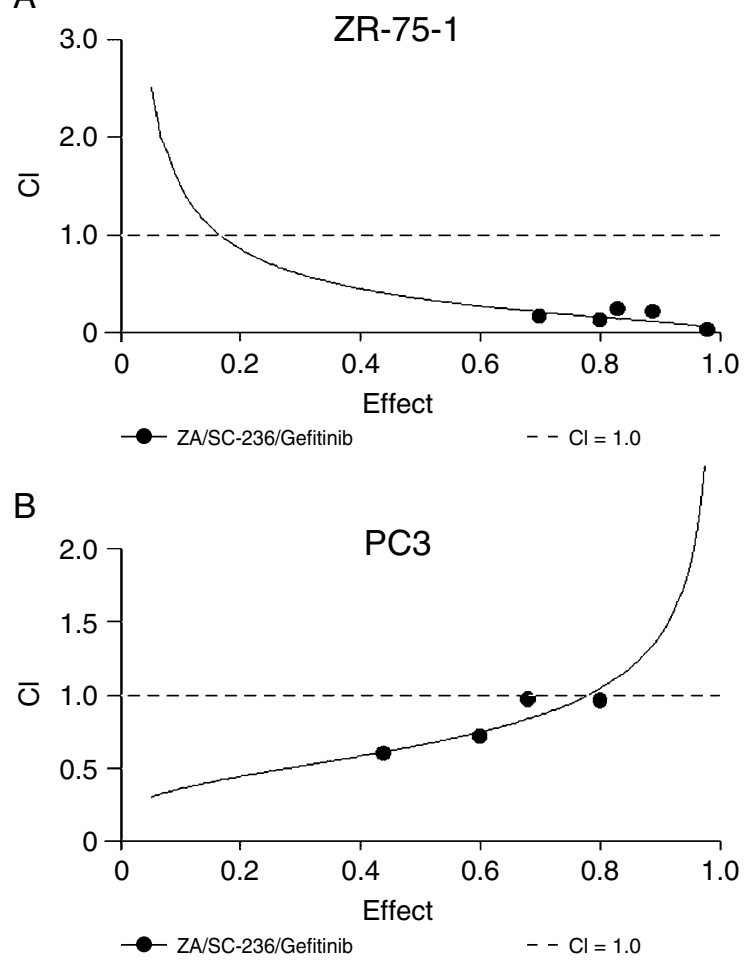

Figure 2 Synergistic effect of ZA, SC-236 and gefitinib in combination on ZR-751 (A) and PC3 (B) cell growth inhibition. The data represent the plot of $\mathrm{Cls}$, a quantitative measure of the degree of drug interaction for a given endpoint of the inhibition effect. The $\mathrm{Cl}$ values of $<1,1$ and $>1$ indicate synergy, additivity and antagonism respectively. Each point is the mean of at least three different replicate experiments.

cooperativity between ZA, SC-236 and gefitinib, we performed a combination analysis at their equipotent ratio and generated $\mathrm{CI}$ and $\mathrm{CI}$-effect plots, according to Chou and Talalay (1984), using an automated calculation software (Fig. 2A and B). With this mathematical model, synergistic conditions occur when the $\mathrm{CI}$ is below 1.0. When the $\mathrm{CI}$ is less than 0.5 , the combination is highly synergistic. Figure $2 \mathrm{~A}$ and $\mathrm{B}$ demonstrates a strong synergism of action of $\mathrm{ZA}$ in combination with SC-236 and gefitinib in PC3 cells $(\mathrm{CI}=0.66)$ and, particularly, in $\mathrm{ZR}$ 75-1 cells $(\mathrm{CI}=0.35)$. The CI-effect plots (Fig. 2A and B) express the quantitative measure of the interaction of the three drugs for a given endpoint of the growth inhibition effect.

\section{Effect of combined treatment on apoptosis}

We evaluated the induction of apoptosis in PC3 and ZR-75-1 cells treated with suboptimal doses of ZA,

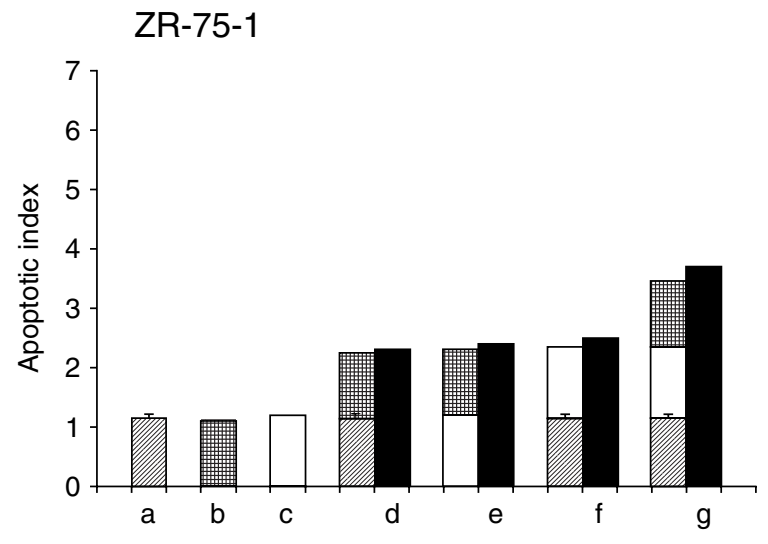

PC3

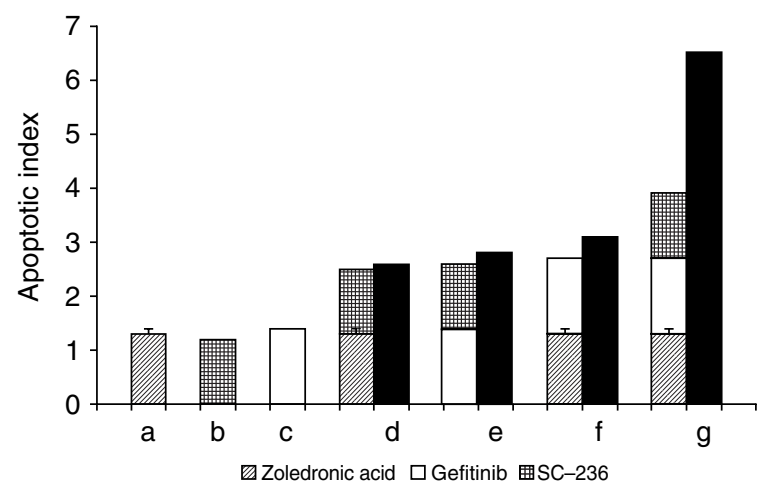

Figure 3 Effect of ZA, SC-236 and gefitinib, alone and in combination, on the induction of apoptosis in ZR-75-1 and PC3 cancer cells. Doses: ZA, $5 \mu \mathrm{M}$ (a, d, f and g); SC-236, $2.5 \mu \mathrm{M}$ (b, d, e and g); gefitinib, $1 \mu \mathrm{M}$ (c, e, f and g). The first three bars show the individual effects of each drug when used alone. The pairs of bars show the effect obtained when the drugs were used in combination: the first of each pair represents, as stacked bars, the expected total effect if the drugs had an additive effect. The second column of each pair (black bar) shows the effect obtained when the drugs were actually used in combination. Data are expressed as the apoptotic index, which represents the ratio between the absorbance of treated cells and that of untreated cells, normalized for the same number of cells. Therefore, results for each treatment are presented relative to control untreated cells, referred to as 1 . Data represent the average of at least two different experiments run in triplicate.

gefitinib and SC-236. As compared with control untreated cells, a mostly additive proapoptotic effect was observed when any two drugs were combined. A clearly supra-additive effect was achieved when the three drugs were combined in PC3 cells, resulting in an apoptotic index over 1.5-fold higher than the sum of each individual agent, while a further additive effect was observed in ZR-75-1 cells as compared with each couple of agents (Fig. 3). 


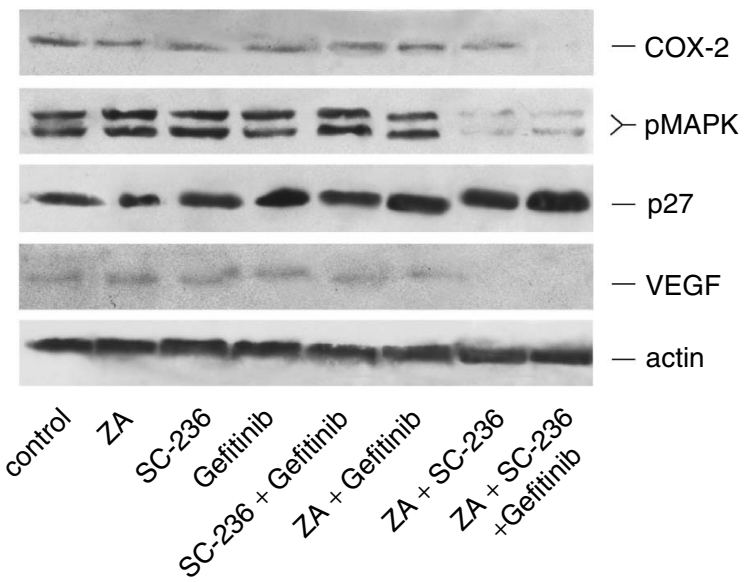

Figure 4 Effect of ZA, SC-236 and gefitinib, alone and in combination, on protein expression. Western blotting analysis of protein expression in ZR-75-1 cells grown in monolayer, untreated or treated for 5 days with ZA $(5 \mu \mathrm{M})$, SC-236 $(2.5 \mu \mathrm{M})$ and gefitinib $(1 \mu \mathrm{M})$ alone or in combination. Cell lysates were processed on day 6 , as described in the Materials and Methods section.

\section{Effect of combined treatment on protein expression}

We evaluated whether any combination treatment could affect the expression of some signaling proteins. As shown in Fig. 4, treatment of ZR-75-1 cells with the combination of ZA $(2.5 \mu \mathrm{M})$ and SC-236 $(1.0 \mu \mathrm{M})$ caused an inhibition of VEGF expression and a decrease of COX-2 and pMAPK expression, while any agent alone, or the combination of ZA+gefitinib, did not cause any change. This effect was further enhanced by addition of gefitinib $(0.5 \mu \mathrm{M})$ to $\mathrm{ZA}+\mathrm{SC}$ 236, resulting in a complete suppression of COX-2 and VEGF expression. In parallel, p27 expression increased with $\mathrm{ZA}+\mathrm{SC}-236$, as compared with single agents or to other coupled treatments, and further increased when the three agents were combined together. Similar data were obtained in PC-3 cells (data not shown).

\section{Effect of combined treatment on tumor growth and protein expression in nude mice}

We next evaluated whether the antitumor effect obtained in vitro with ZA, gefitinib and SC-236 could also be reproduced in vivo in nude mice bearing PC3 tumor xenografts. Groups of 8 mice with tumors of about $0.2 \mathrm{~cm}$ were randomized to receive suboptimal doses of ZA, gefitinib and SC-236, alone and in combination, to evaluate the occurrence of any cooperative effect among these drugs.

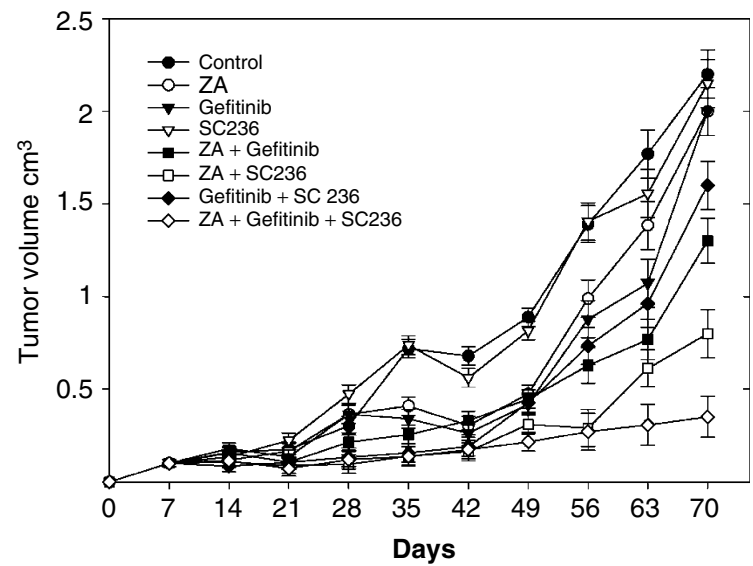

Figure 5 Antitumor activity of ZA treatment in combination with SC-236 and gefitinib on established PC3 human prostate carcinoma xenografts in nude mice. Mice were injected s.c. in the dorsal flank with ten PC3 cells. After 7 days (average tumor size, $0.2-0.3 \mathrm{~cm}^{3}$ ), mice were treated i.p. as described in the Materials and Methods section. The doses of each drug, used alone or in combination, were as follows: ZA, $400 \mathrm{mg} / \mathrm{kg}$ i.p.; SC-236, $12 \mathrm{mg} / \mathrm{kg}$ i.p.; gefitinib, $150 \mathrm{mg} / \mathrm{kg}$ i.p. Data represent the average $\pm S D$.

As shown in Fig. 5, treatment with SC-236 had only a modest inhibitory effect, while treatments with single-agent ZA or gefitinib transiently inhibited tumor growth, although tumors resumed a growth rate similar to that of untreated mice upon cessation of treatment. In contrast, in animals treated with any two agents, particularly with the ZA+SC-236 combination, an additive antitumor effect causing tumor growth delay was obtained. A dramatic and sustained inhibitory effect was obtained when the three drugs were combined together. In fact, only a modest increase in tumor size was recorded at the end of the experiment, 10 weeks after tumor cell injection and 5 weeks after treatment withdrawal. This effect was accompanied by a relevant prolongation of survival in this combination therapy group, as compared with other groups. The combined treatment with any two drugs or with all three drugs together was well tolerated; no weight loss or other signs of acute or delayed toxicity were observed.

In order to understand the mechanism of action underlying the in vivo activity of the combinations, we evaluated whether expression of some signaling proteins could be affected by any treatments. As represented in Fig. 6, western blot analysis of PC3 tumor specimens removed at the end of treatment, while they did not demonstrate any effects on the expression of Ras and COX-2, revealed a remarkable modulation of VEGF expression. In fact, as described 


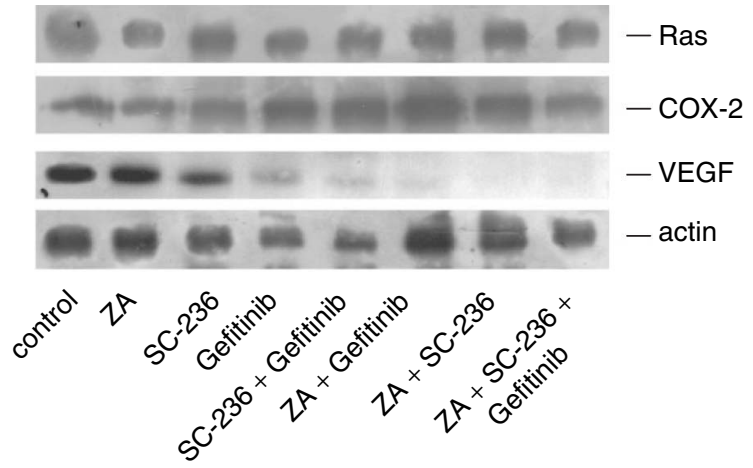

Figure 6 Ras, COX-2 and VEGF expression in tumor specimens removed from mice treated with the different agents at the end of treatment. Western blotting analysis was carried out as described in the Materials and Methods section.

also in vitro, VEGF expression showed only a moderate reduction with any single agent, a more marked inhibition with two agents in combination, resulting in complete suppression with $\mathrm{ZA}+\mathrm{SC}-236$ as well as with the three agents together.

\section{Discussion}

Recent studies are highlighting the ability of certain novel drugs, originally not devised as antitumor agents, to interfere with the function/expression of signalling proteins that play a critical role in the processes of tumor growth and spread. BPs are widely used to inhibit osteoclastic activity in malignant diseases involving excessive bone resorption such as metastatic prostate and breast carcinoma (Adami 1997; Hillner et al. 2003). Recent experimental evidence has demonstrated an anticancer effect of $\mathrm{BPs}$, such as ZA, through the direct inhibition of tumor cell proliferation, survival, migration and invasion, and the suppression of angiogenesis (Green 2003). At molecular level, the main targets of ZA action have recently been found to be the farnesylpyrophosphate and/or geranylgeranylpyrophosphate synthetase, leading to decreased generation of farnesyl diphosphate and geranylgeranyl diphosphate, isoprenoid intermediates required for post-translational prenylation of key small GTP-binding proteins such as Ras and Rho, essential for many cellular functions (Rogers et al. 2000).

The enzyme COX-2 is overexpressed in a variety of malignancies, including breast (Parrett et al. 1997) and prostate cancer (Gupta et al. 2000), and is associated with increased malignant potential, probably through the activity of COX-2-derived PGs (Turini \& DuBois 2002). Crosstalk between PG receptors and the EGFR may also contribute to increased cell growth (Pai et al. 2002; Buchanan et al. 2003; Shao et al. 2003). On the other hand, it has been demonstrated that COX-2 expression is transcriptionally induced by the Rasmediated signaling cascade triggered by EGFR activation (Turini \& DuBois 2002). Further evidence of a functional interaction between EGFR and COX-2 is provided by our recent demonstration of a cooperative antitumor and antiangiogenic effect obtained with the combination of selective EGFR and COX-2 inibitors (Tortora et al. 2003).

Due to these intimate functional interactions, in this study we have used the new generation N-BP, ZA, the small molecule selective EGFR tyrosine kinase inhibitor, gefitinib, and the COX-2 inhibitor, SC-236; hypothesizing that the combination of these noncytotoxic agents, by interfering with key and complementary pathways involved in tumor growth and angiogenesis, may result in an antitumor effect.

Based on previous observations of antitumor activity of the combination of gefitinib and a COX-2 inhibitor (Tortora et al. 2003, Chen et al. 2004) we demonstrated that both gefitinib and SC-236 in combination with ZA show a cooperative growth inhibitory effect on the in vitro soft agar growth of PC3 prostate cancer and ZR-75-1 breast cancer cells (Fig. 1). The most potent effect obtained with any couple was achieved by ZA+SC-236, while a synergistic $(\mathrm{CI}=0.66)$ and a strongly synergistic $(\mathrm{CI}=0.35)$ effect was achieved in PC3 and in ZR 75-1 cell lines when all three agents were combined, as demonstrated using the CI model (Fig. 2A and B).

There is increasing experimental evidence indicating that BPs may exert anticancer effects through the direct inhibition of tumor cell survival (Tassone et al. 2003). We demonstrated that the effect of combination treatment on tumor cells was not simply cytostatic, as the result of inhibition of tumor cell proliferation, but might be due to an increase in apoptotic cell death (Fig. 3).

The MAPK pathway is implicated in growth control in all its facets, including cell proliferation, transformation, differentiation and apoptosis. Although formation of tumor vessels is a complex and coordinated process, signalling by VEGF represents a crucial ratelimiting step in pathological angiogenesis during tumor progression (Tortora et al. 2004). Moreover, we and other groups have linked both VEGF and COX-2 to the development of resistance to the antitumor activity of EGFR inhibitors (Viloria-Petit et al. 2001; Ciardiello et al. 2004). p27, a cyclin-dependent kinase inhibitor, directs the timely exit of cells from the cell cycle in response to antimitogenic signals (Blain et al. 
2003). In order to find a potential mechanism of action underlying the therapeutic activity of the three-drug combination we evaluated whether any combination treatment could affect the expression of these proteins. We have found that the antiproliferative effect of the combined treatment was accompanied by a cooperative down-regulation of the expression of COX-2, pMAPK and VEGF expression, whilst p27 expression increased.

We next evaluated whether the cooperative effect obtained in vitro could also be reproduced in vivo in nude mice bearing PC3 human prostate cancer xenografts. We used suboptimal doses of ZA, SC-236 and gefitinib to determine any cooperative effect among these drugs. Treatment with single-agent ZA transiently inhibits tumor growth because tumors resume the growth rate of untreated control after cessation of treatment. In contrast, we showed that any two agents used in combination may cooperate in delaying tumor growth, particularly ZA+SC-236. A marked cooperative effect was obtained when the three agents were used together.

We have analyzed whether a modulation of the expression of Ras and other proteins could be involved in this effect. Interestingly, no changes were detectable in Ras expression as well as in COX-2 expression, while, as observed in vitro, a potent inhibition of VEGF expression was caused by ZA+SC-236 and a complete suppression was obtained with the three agents together.

Recently, it has been reported that ZA impairs the EGFR-dependent activation of the key signal transducers MAPK and Akt, therefore interfering with the early steps of the EGFR-activated pathways (Caraglia et al. 2004). This activity may explain in part our observation that, although the addition of gefitinib can add a further inhibitory effect, the combination of $\mathrm{ZA}+\mathrm{SC}-236$ is already remarkably active on tumor growth and protein expression, partly shadowing the contribution of the selective EGFR inhibitor.

Several recent studies have demonstrated the antitumor activity of ZA in vitro (Matsumoto et al. 2005) as well as in in vivo orthotopical bone models of prostate and breast cancer, also in combination with protein tyrosine kinase inhibitor and cytotoxic drugs (Kim et al. 2005) This is the first study demonstrating that a relevant cooperative antitumor effect can be achieved in an in vivo non-bone metastastatic model by combining ZA with SC-236 and gefitinib. Moreover, the results show that this cooperative effect can produce sustained control of cancer growth, inducing apoptosis and affecting the expression of relevant proteins involved in cell proliferation and angiogenesis.
The efficacy of ZA in the management of bone metastatic breast and prostate cancer has led to investigations into its potential antitumor activity in non bone metastatic patients and, in this regard, several studies are ongoing. We believe that our study provides a further insight into the antitumor effect of $\mathrm{ZA}$ in combination with selective signal transducers, supporting a treatment strategy that could be translated in a clinical setting.

\section{Funding}

This study was supported by a national grant from the Associazione Italiana per la Ricerca sul Cancro (AIRC) and regional grant AIRC Regione Campania, the CNR-MIUR 449/97-99, the Ministry of Health RF02 and the Centro di Competenza Regionale 'Genomics for Applied Research' (GEAR). The authors declare that there is no conflict of interest that would prejudice the impartiality of this scientific work.

\section{References}

Adami S 1997 Biphosphonate in prostate carcinoma. Cancer 80 1674-1679.

Blain SW, Scher HI, Cordon-Cardo C \& Koff A 2003 p27 as a target for cancer therapeutics. Cancer Cell 3 111-115.

Buchanan FG, Wang D, Bargiacchi F \& DuBois RN 2003 Prostaglandin E2 regulates cell migration via the intracellular activation of the epidermal growth factor receptor. Journal of Biological Chemistry 278 35451-35457.

Caraglia M, D'Alessandro AM, Marra M, Giuberti G, Vitale G, Viscomi C, Colao A, Prete SD, Tagliaferri P, Tassone $\mathrm{P}$ et al. 2004 The farnesyl transferase inhibitor R115777 (Zarnestra) synergistically enhances growth inhibition and apoptosis induced on epidermoid cancer cells by Zoledronic acid (Zometa) and Pamidronate. Oncogene 23 6900-6913.

Chen Z, Zhang X, Li M, Wang Z, Wieand HS, Grandis JR \& Shin DM 2004 Simultaneously targeting epidermal growth factor receptor tyrosine kinase and cyclooxygenase-2, an efficient approach to inhibition of squamous cell carcinoma of the head and neck. Clinical Cancer Research 10 5930-5939.

Chou TC \& Talalay P 1984 Quantitative analysis of doseeffect relationships: the combined effects of multiple drugs or enzyme inhibitors. Advances in Enzyme Regulation 22 27-55.

Ciardiello F \& Tortora G 2001 A novel approach in the treatment of cancer: targeting the epidermal growth factor receptor. Clinical Cancer Research 7 2958-2970.

Ciardiello F, Damiano V, Bianco R, Bianco C, Fontanini G, De Laurentiis M, De Placido S, Mendelsohn J, Bianco AR \& Tortora G 1996 Antitumor activity of combined blockade of epidermal growth factor receptor and protein 
kinase A. Journal of the National Cancer Institute (Bethesda) 88 1770-1776.

Ciardiello F, Bianco R, Caputo R, Damiano V, Troiani T, Melisi D, De Vita F, De Placido S, Bianco AR \& Tortora G 2004 Antitumor activity of ZD6474, a vascular endothelial growth factor receptor tyrosine kinase inhibitor, in human cancer cells with acquired resistance to antiepidermal growth factor receptor therapy. Clinical Cancer Research 10 784-793.

Green JR 2003 Antitumor effects of bisphosphonates. Cancer 97 840-847.

Gupta S, Srivastava M, Ahmad N, Bostwick DG \& Mukhtar H 2000 Over-expression of cyclooxygenase-2 in human prostate adenocarcinoma. Prostate 42 73-78.

Hillner BE, Ingle JN, Chlebowski RT, Gralow J, Yee GC, Janjan NA, Cauley JA, Blumenstein BA, Albain KS, Lipton A et al. 2003 American Society of Clinical Oncology 2003 update on the role of bisphosphonates and bone health issues in women with breast cancer. Journal of Clinical Oncology 21 4042-4057.

Kim SJ, Uehara H, Yazici S, He J, Langley RR, Mathew P, Fan D \& Fidler IJ 2005 Modulation of bone microenvironment with zoledronate enhances the therapeutic effects of STI571 and paclitaxel against experimental bone metastasis of human prostate cancer. Cancer Research 65 3707-3715.

Lacerna L \& Hohneker J 2003 Zoledronic acid for the treatment of bone metastases in patients with breast cancer and other solid tumors. Seminars in Oncology 30 150-160.

Masferrer JL, Koki A \& Seibert K 1999 COX-2 inhibitors. A new class of antiangiogenic agents. Annals of the New York Academy of Sciences $\mathbf{8 8 9} 84-86$.

Matsumoto S, Kimura S, Segawa H, Kuroda J, Yuasa T, Sato K, Nogawa M, Tanaka F, Maekawa T \& Wada H 2005 Efficacy of the third-generation bisphosphonate, zoledronic acid alone and combined with anti-cancer agents against small cell lung cancer cell lines. Lung Cancer 47 31-39.

Mendelsohn J \& Baselga J 2003 Status of epidermal growth factor receptor antagonists in the biology and treatment of cancer. Journal of Clinical Oncology 21 2787-2789.

Pai R, Soreghan B, Szabo IL, Pavelka M, Batar D \& Tarnawski AS 2002 Prostaglandin E2 transactivates EGF receptor: a novel mechanism for promoting colon cancer growth and gastrointestinal hypertrophy. Nature Medicine 8 289-293.
Parrett ML, Harris RE, Joarder FS, Ross MS, Clausen KP \& Robertson FM 1997 Cyclooxygenase-2 gene expression in human breast cancer. International Journal of Oncology $\mathbf{1 0}$ 503-507.

Rogers MJ, Gordon S, Benford HL, Coxon FP, Luckman SP, Monkkonen J \& Frith JC 2000 Cellular and molecular mechanisms of action of bisphosphonates. Cancer $\mathbf{8 8}$ 2961-2978.

Shao J, Lee SB, Guo H, Evers BM \& Sheng H 2003 Prostaglandin E2 stimulates the growth of colon cancer cells via induction of amphiregulin. Cancer Research $\mathbf{6 3}$ 5218-5223.

Sinensky M 2000 Recent advances in the study of prenylated proteins. Biochimica et Biophysica Acta 1484 93-106.

Smith WL, DeWitt DL \& Garavito RM 2000 Cyclooxygenases: structural, cellular, and molecular biology. Annual Review of Biochemistry 69 145-182.

Tassone P, Tagliaferri P, Viscomi C, Palmieri C, Caraglia M, D'Alessandro A, Galea E, Goel A, Abbruzzese A, Boland CR et al. 2003 Zoledronic acid induces antiproliferative and apoptotic effects in human pancreatic cancer cells in vitro. British Journal of Cancer 88 1971-1978.

Tortora G, Damiano V, Bianco C, Baldassarre G, Bianco AR, Lanfrancone L, Pelicci PG \& Ciardiello F 1997 The RI subunit of protein kinase A (PKA) binds to Grb2 and allows PKA interaction with the activated EGF-receptor. Oncogene 14 923-928.

Tortora G, Caputo R, Damiano V, Melisi D, Bianco R, Fontanini G, Veneziani BM, De Placido S, Bianco AR \& Ciardiello F 2003 Combination of a selective cyclooxygenase- 2 inhibitor with epidermal growth factor receptor tyrosine kinase inhibitor ZD1839 and protein kinase A antisense causes cooperative antitumor and antiangiogenic effect. Clinical Cancer Research 9 1566-1572.

Tortora G, Melisi D \& Ciardiello F 2004 Angiogenesis: a target for cancer therapy. Current Pharmaceutical Design 10 11-26.

Turini ME \& DuBois RN 2002 Cyclooxygenase-2: a therapeutic target. Annual Review of Medicine 53 35-57.

Viloria-Petit A, Crombet T, Jothy S, Hicklin D, Bohlen P, Schlaeppi JM, Rak J \& Kerbel RS 2001 Acquired resistance to the antitumor activity of epidermal growth factor receptor-blocking antibodies in vivo: a role for altered tumor angiogenesis. Cancer Research $\mathbf{6 1}$ 5090-5101. 